\title{
Time-dependent Maxwell field operators and field energy density for an atom near a conducting wall
}

\author{
Ruggero Vasile, ${ }^{1}$ Riccardo Messina,${ }^{2,3}$ and Roberto Passante ${ }^{2}$ \\ ${ }^{1}$ Department of Physics and Astronomy, University of Turku, 20014 Turun Yliopisto, Finland \\ ${ }^{2}$ Dipartimento di Scienze Fisiche e Astronomiche dell'Universita' degli \\ Studi di Palermo and CNSIM, Via Archirafi 36, I-90123 Palermo, Italy \\ ${ }^{3}$ Laboratoire Kastler Brossel, case 74, CNRS, ENS, \\ UPMC, Campus Jussieu, F-75252 Paris Cedex 05, France
}

(Dated: December 23, 2018)

\begin{abstract}
We consider the time evolution of the electric and magnetic field operators for a two-level atom, interacting with the electromagnetic field, placed near an infinite perfectly conducting wall. We solve iteratively the Heisenberg equations for the field operators and obtain the electric and magnetic energy density operators around the atom (valid for any initial state). Then we explicitly evaluate them for an initial state with the atom in its bare ground state and the field in the vacuum state. We show that the results can be physically interpreted as the superposition of the fields propagating directly from the atom and the fields reflected on the wall. Relativistic causality in the field propagation is discussed. Finally we apply these results to the calculation of the dynamical Casimir-Polder interaction energy in the far zone between two atoms when a boundary condition such as a conducting wall is present. Magnetic contributions to the interatomic Casimir-Polder interaction in the presence of the wall are also considered. We show that, in the limit of large times, the known results of the stationary case are recovered.
\end{abstract}

PACS numbers: 12.20.Ds, 42.50.Ct

\section{INTRODUCTION}

The existence of zero-point field fluctuations in quantum electrodynamics has striking consequences such as Lamb shift, spontaneous decay, Casimir and CasimirPolder forces, which give evidence of the quantum nature of the electromagnetic field [1, 2]. Moreover, Casimir forces are a manifestation of the quantum properties of the radiation field and of vacuum fluctuations at the macroscopic level. Vacuum fluctuations are modified by the presence of boundary conditions as well as by atoms or molecules. Thus the presence of macroscopic objects can change the radiative properties of the atoms. In particular, it is known that long-range intermolecular forces change when the atoms are in the proximity of a boundary condition such as a conducting plate or a cavity [3, 4]. This is also important from an experimental point of view, because a precise comparison between measurements of Casimir and Casimir-Polder forces requires calculating these forces in situations as close as possible to realistic laboratory situations. Also, it has been recently suggested that appropriate boundary conditions may enhance Casimir-Polder forces [5, 6, 7,, 8$]$, and this can be very important for their direct experimental measurement (usually they are very tiny forces).

Calculation of time-dependent electric and magnetic field operators in the Heisenberg picture generated by an atom, even when specific boundary conditions are present, are a powerful and general tool to calculate various radiative processes for different initial atomic states. This kind of calculation have been done some years ago by Power and Thirunamachandran for atoms in the free space [9]; they have also successfully applied the results for the evaluation of intermolecular CasimirPolder forces, both for ground-state and excited-state atoms [10, 11, 12].

In this paper, following the same method of Power and Thirunamachandran, we calculate the time-dependent electric and magnetic Maxwell field operators in the Heisenberg representation generated by an atom placed near an infinite and perfectly reflecting plate. We will also apply our results to the evaluation of the dynamical (time-dependent) Casimir-Polder interaction between two neutral atoms near a conducting wall. We shall consider both electric and magnetic interactions. Dynamical Casimir-Polder interactions have been recently considered for atoms in the free space also for partially dressed states [13] or excited states [14], as well as in the case of one single atom and an infinite conducting wall [15, 16, 17]. The method used is very suitable for generalization to other initial states of the system.

This paper is organized as follows. In Section [I] we introduce the multipolar coupling model for a two-level atom interacting with the radiation field in the electric dipole approximation and in the presence of a conducting wall. Then we solve the Heisenberg equations for the photon creation and annihilation operators up to the second order in the electric charge, using an iterative technique. In Sections III we evaluate first- and second-order corrections to the electric and magnetic field operators, discussing their physical properties. Finally, in Sec. IV we use the results obtained to evaluate the electric and magnetic energy density operators and their expectation values on the bare atom-field ground state. In Section V] we use the expressions obtained for discussing dynamical electric and magnetic Casimir-Polder interaction 
energies between two atoms or polarizable bodies when a boundary conditions such as the conducting plate is present. We remark that the expressions obtained for the perturbative expansion of the fields are general and do not depend on the specific initial state of the atomfield systems. Hence they can be also used for evaluating Casimir-Polder energies for different initial states such as partially dressed or excited states.

\section{THE HAMILTONIAN MODEL}

We consider a two-level atom in front of an infinite and perfectly conducting wall, interacting with the quan- tum electromagnetic radiation field. We choose a reference frame such that the atom is placed in $\mathbf{r}_{A} \equiv(0,0, d)$ whereas the mirror coincides with the plane $z=0$. We work in the multipolar coupling scheme and within the electric dipole approximation [18, 19]. Thus the Hamiltonian describing our system reads

$$
\begin{aligned}
H & =H_{0}+H_{I} \\
H_{0} & =\hbar \omega_{0} S_{z}+\sum_{\mathbf{k} j} \hbar \omega_{\mathbf{k}} a_{\mathbf{k} j}^{\dagger} a_{\mathbf{k} j} \\
H_{I} & =-i \sqrt{\frac{2 \pi \hbar c}{V}} \sum_{\mathbf{k} j} \sqrt{k}\left(\boldsymbol{\mu} \cdot \mathbf{f}_{\mathbf{k} j}\left(\mathbf{r}_{A}\right)\right)\left(a_{\mathbf{k} j}-a_{\mathbf{k} j}^{\dagger}\right)\left(S_{+}+S_{-}\right) .
\end{aligned}
$$

In this expression the field is described by the set of bosonic annihilation and creation operators $a_{\mathbf{k} j}$ and $a_{\mathbf{k} j}^{\dagger}$, associated with a photon of frequency $\omega_{\mathbf{k}}=c k$, while the matrix element of the electric dipole moment operator $\boldsymbol{\mu}$ and the pseudospin operators $S_{+}, S_{-}$and $S_{z}$ are associated to the atom, which has a transition frequency $\omega_{0}$
2]. Also, $\mathbf{f}_{\mathbf{k} j}(\mathbf{r})$ are the field mode functions in the presence of the wall, which in Eq. (11) are evaluated at the atomic position $\mathbf{r}_{\mathbf{A}}$. Their expressions can be obtained from the mode functions of a perfectly conducting cubical cavity of volume $V=L^{3}$ with walls $(-L / 2<x, y<L / 2$, $0<z<L)[1,[3]$

$$
\begin{aligned}
\left(\mathbf{f}_{\mathbf{k} j}(\mathbf{r})\right)_{x} & =\sqrt{8}\left(\hat{e}_{\mathbf{k} j}\right)_{x} \cos \left[k_{x}\left(x+\frac{L}{2}\right)\right] \sin \left[k_{y}\left(y+\frac{L}{2}\right)\right] \sin \left(k_{z} z\right) \\
\left(\mathbf{f}_{\mathbf{k} j}(\mathbf{r})\right)_{y} & =\sqrt{8}\left(\hat{e}_{\mathbf{k} j}\right)_{y} \sin \left[k_{x}\left(x+\frac{L}{2}\right)\right] \cos \left[k_{y}\left(y+\frac{L}{2}\right)\right] \sin \left(k_{z} z\right) \\
\left(\mathbf{f}_{\mathbf{k} j}(\mathbf{r})\right)_{z} & =\sqrt{8}\left(\hat{e}_{\mathbf{k} j}\right)_{z} \sin \left[k_{x}\left(x+\frac{L}{2}\right)\right] \sin \left[k_{y}\left(y+\frac{L}{2}\right)\right] \cos \left(k_{z} z\right)
\end{aligned}
$$

where $k_{x}=l \pi / L, k_{y}=m \pi / L, k_{z}=n \pi / L(l, m, n=$ $0,1,2, \ldots)$ and $\hat{e}_{\mathbf{k} j}$ are polarization unit vectors. In order to switch from the cavity to the wall at $z=0$ one has to take, at the end of the calculations, the limit $L \rightarrow \infty$.

As discussed in the Introduction, many properties of the quantum electromagnetic field and of the atom, in the presence of the wall, can be studied in terms of the expressions of the time-dependent electric and magnetic field operators for the interacting system. In the Heisen- berg picture they can be written as

$$
\begin{aligned}
& \mathbf{E}(\mathbf{r}, t)=i \sqrt{\frac{2 \pi \hbar c}{V}} \sum_{\mathbf{k} j} \sqrt{k} \mathbf{f}_{\mathbf{k} j}(\mathbf{r}) a_{\mathbf{k} j}(t)+\text { h.c. } \\
& \mathbf{H}(\mathbf{r}, t)=\sqrt{\frac{2 \pi \hbar c}{V}} \sum_{\mathbf{k} j} \sqrt{\frac{1}{k}}\left[\nabla \times \mathbf{f}_{\mathbf{k} j}(\mathbf{r})\right] a_{\mathbf{k} j}(t)+\text { h.c. }
\end{aligned}
$$

where the time dependence is fully included in the photon creation and annihilation operators. Their expressions are formally given by the solutions of the relative Heisenberg equations associated to our Hamiltonian op- 
erator (11). However, it is not possible to solve exactly these equations for our model. Thus we use an iterative technique and the solution for the annihilation operator can be written as a power series in the coupling constant

$$
a_{\mathbf{k} j}(t)=a_{\mathbf{k} j}^{(0)}(t)+a_{\mathbf{k} j}^{(1)}(t)+a_{\mathbf{k} j}^{(2)}(t)+\ldots
$$

where the contribution $a_{\mathbf{k} j}^{(i)}(t)$ is proportional to the $i$-th power of the electric charge. By taking the Hermitian conjugate we get the analogous expansion for the creation operator. For our purposes we need their expressions up to the the second order only. The result, which is a straightforward generalization to the case with the boundary condition of that obtained for an atom in the free space [9, 14], is

$$
\begin{aligned}
a_{\mathbf{k} j}^{(0)}(t)= & e^{-i \omega_{\mathbf{k}} t} a_{\mathbf{k} j} \\
a_{\mathbf{k} j}^{(1)}(t)= & e^{-i \omega_{\mathbf{k}} t} \sqrt{\frac{2 \pi c k}{\hbar V}}\left(\boldsymbol{\mu} \cdot \mathbf{f}_{\mathbf{k} j}\left(\mathbf{r}_{A}\right)\right)\left[S_{+} F\left(\omega_{\mathbf{k}}+\omega_{0}, t\right)+S_{-} F\left(\omega_{\mathbf{k}}-\omega_{0}, t\right)\right] \\
a_{\mathbf{k} j}^{(2)}(t)= & e^{-i \omega_{\mathbf{k}} t} \frac{4 \pi i c}{\hbar V} S_{z}\left(\boldsymbol{\mu} \cdot \mathbf{f}_{\mathbf{k} j}\left(\mathbf{r}_{A}\right)\right) \sum_{\mathbf{k}^{\prime} j^{\prime}} \sqrt{k k^{\prime}}\left(\boldsymbol{\mu} \cdot \mathbf{f}_{\mathbf{k}^{\prime} j^{\prime}}\left(\mathbf{r}_{A}\right)\right) \\
& \times\left[a_{\mathbf{k}^{\prime} j^{\prime}}\left(\frac{F\left(\omega_{0}+\omega_{\mathbf{k}}, t\right)-F\left(\omega_{\mathbf{k}}-\omega_{\mathbf{k}^{\prime}}, t\right)}{\omega_{0}+\omega_{\mathbf{k}^{\prime}}}+\frac{F\left(\omega_{\mathbf{k}}-\omega_{0}, t\right)-F\left(\omega_{\mathbf{k}}-\omega_{\mathbf{k}^{\prime}}, t\right)}{\omega_{0}-\omega_{\mathbf{k}^{\prime}}}\right)\right. \\
& \left.-a_{\mathbf{k}^{\prime} j^{\prime}}^{\dagger}\left(\frac{F\left(\omega_{0}+\omega_{\mathbf{k}}, t\right)-F\left(\omega_{\mathbf{k}}+\omega_{\mathbf{k}^{\prime}}, t\right)}{\omega_{0}-\omega_{\mathbf{k}^{\prime}}}+\frac{F\left(\omega_{\mathbf{k}}-\omega_{0}, t\right)-F\left(\omega_{\mathbf{k}}+\omega_{\mathbf{k}^{\prime}}, t\right)}{\omega_{0}+\omega_{\mathbf{k}^{\prime}}}\right)\right]
\end{aligned}
$$

where we have introduced the auxiliary function

$$
F(\omega, t)=\int_{0}^{t} e^{i \omega t^{\prime}} d t^{\prime}=\frac{e^{i \omega t}-1}{i \omega}
$$

All the operators appearing in the RHS of Eq. (5) are evaluated at $t=0$ : from now onwards, when atomic and photonic operators are written without an explicit time dependence, it is meant they are at $t=0$. The zeroth-order term does not depend on the presence of the source, being only the free-field contribution. As in the case of an atom in the free space discussed in [9], the first order term contains only atomic operators, and the second order term contains both photonic and atomic operators. Substituting the expansions (44) and (5) into (3), we get the iterative expansion of the electric and magnetic Maxwell field operators

$$
\begin{aligned}
& \mathbf{E}(\mathbf{r}, t)=\mathbf{E}^{(0)}(\mathbf{r}, t)+\mathbf{E}^{(1)}(\mathbf{r}, t)+\mathbf{E}^{(2)}(\mathbf{r}, t)+\ldots \\
& \mathbf{H}(\mathbf{r}, t)=\mathbf{H}^{(0)}(\mathbf{r}, t)+\mathbf{H}^{(1)}(\mathbf{r}, t)+\mathbf{H}^{(2)}(\mathbf{r}, t)+\ldots
\end{aligned}
$$

In the next Section we explicitly evaluate the first and second order corrections of the fields.

\section{PERTURBATIVE EXPANSION OF THE FIELD OPERATORS}

Using the first of (5), the expression of the zeroth-order term is

$$
\begin{aligned}
& \mathbf{E}^{(0)}(\mathbf{r}, t)=i \sqrt{\frac{2 \pi \hbar c}{V}} \sum_{\mathbf{k} j} \sqrt{k} \mathbf{f}_{\mathbf{k} j}(\mathbf{r}) e^{-i \omega_{\mathbf{k}} t} a_{\mathbf{k} j}+\text { h.c. } \\
& \mathbf{H}^{(0)}(\mathbf{r}, t)=\sqrt{\frac{2 \pi \hbar c}{V}} \sum_{\mathbf{k} j} \sqrt{\frac{1}{k}}\left[\nabla \times \mathbf{f}_{\mathbf{k} j}(\mathbf{r})\right] e^{-i \omega_{\mathbf{k}} t} a_{\mathbf{k} j}+\text { h.c. }
\end{aligned}
$$

In order to obtain the first-order correction, we substitute the second of (5) into (3), obtaining

$$
\begin{aligned}
& \mathbf{E}^{(1)}(\mathbf{r}, t)=\frac{2 \pi i c}{V} \sum_{\mathbf{k} j} k \mathbf{f}_{\mathbf{k} j}(\mathbf{r})\left(\boldsymbol{\mu} \cdot \mathbf{f}_{\mathbf{k} j}\left(\mathbf{r}_{A}\right)\right) \\
& \times\left(S_{+} F\left(\omega_{0}+\omega_{\mathbf{k}}, t\right)+S_{-} F\left(\omega_{\mathbf{k}}-\omega_{0}, t\right)\right) e^{-i \omega_{\mathbf{k}} t}+\text { h.c. } \\
& \mathbf{H}^{(1)}(\mathbf{r}, t)=\frac{2 \pi c}{V} \sum_{\mathbf{k} j}\left[\nabla \times \mathbf{f}_{\mathbf{k} j}(\mathbf{r})\right]\left(\boldsymbol{\mu} \cdot \mathbf{f}_{\mathbf{k} j}\left(\mathbf{r}_{A}\right)\right) \\
& \times\left(S_{+} F\left(\omega_{0}+\omega_{\mathbf{k}}, t\right)+S_{-} F\left(\omega_{\mathbf{k}}-\omega_{0}, t\right)\right) e^{-i \omega_{\mathbf{k}} t}+\text { h.c. }
\end{aligned}
$$

We can now take the continuum limit, by allowing $L \rightarrow \infty$. The continuum limit can be performed with the following prescription $\sum_{\mathbf{k} j} \rightarrow \frac{V}{(2 \pi)^{3}} \sum_{j} \int d^{3} \mathbf{k}$. The mode 
functions $\mathbf{f}_{\mathbf{k} j}(\mathbf{r})$ are of course affected by this transformation since they contain $L$. The result for the $p=x, y, z$ component of the fields is

$$
\begin{aligned}
& E_{p}^{(1)}(\mathbf{r}, t)=\frac{i c}{4 \pi^{2}} \sum_{q} \mu_{q} \int d k k^{3}\left(S_{+} F\left(\omega_{0}+\omega_{\mathbf{k}}, t\right)+S_{-} F\left(\omega_{\mathbf{k}}-\omega_{0}, t\right)\right) e^{-i \omega_{\mathbf{k}} t} \sum_{j} \int d \Omega\left(\mathbf{f}_{\mathbf{k} j}(\mathbf{r})\right)_{p}\left(\mathbf{f}_{\mathbf{k} j}\left(\mathbf{r}_{A}\right)\right)_{q} \\
& H_{p}^{(1)}(\mathbf{r}, t)=\frac{c}{4 \pi^{2}} \sum_{q} \mu_{q} \int d k k^{2}\left(S_{+} F\left(\omega_{0}+\omega_{\mathbf{k}}, t\right)+S_{-} F\left(\omega_{\mathbf{k}}-\omega_{0}, t\right)\right) e^{-i \omega_{\mathbf{k}} t} \sum_{j} \int d \Omega\left(\nabla \times \mathbf{f}_{\mathbf{k} j}(\mathbf{r})\right)_{p}\left(\mathbf{f}_{\mathbf{k} j}\left(\mathbf{r}_{A}\right)\right)_{q}
\end{aligned}
$$

where the integrations over $k$ run from $-\infty$ to $+\infty, \mu_{q}$ is the $q=x, y, z$ component of $\boldsymbol{\mu}$. The polarization sum and the integral over angular variables can be explicitly calculated by lengthy straightforward calculations (see the Appendix for more details). Finally, we get

$$
\begin{aligned}
& E_{p}^{(1)}(\mathbf{r}, t)=\frac{i c}{\pi} \sum_{q} \sum_{\nu=0,1}(-1)^{\nu\left(1-\delta_{q z}\right)} \mu_{q} F_{p q} \frac{1}{r_{\nu}} \int d k\left(S_{+} F\left(\omega_{0}+\omega_{\mathbf{k}}, t\right)+S_{-} F\left(\omega_{\mathbf{k}}-\omega_{0}, t\right)\right) e^{-i \omega_{\mathbf{k}} t} \sin \left(k r_{\nu}\right) \\
& H_{p}^{(1)}(\mathbf{r}, t)=\frac{c}{\pi} \sum_{q} \sum_{\nu=0,1}(-1)^{\nu\left(1-\delta_{q z}\right)} \mu_{q} G_{p q} \frac{1}{r_{\nu}} \int d k k\left(S_{+} F\left(\omega_{0}+\omega_{\mathbf{k}}, t\right)+S_{-} F\left(\omega_{\mathbf{k}}-\omega_{0}, t\right)\right) e^{-i \omega_{\mathbf{k}} t} \sin \left(k r_{\nu}\right)
\end{aligned}
$$

where $F_{p q}=-\nabla^{2} \delta_{p q}+\nabla_{p} \nabla_{q}, G_{p q}=-\epsilon_{p q s} \nabla_{s}\left(\epsilon_{\alpha \beta \gamma}\right.$ being the complete antisymmetric tensor), and $\nabla_{p} \equiv \frac{\partial}{\partial x_{p}}$ $(p=1,2,3)$ are differential operators acting on the variables $x, y, z$. The index $\nu=0,1$ labels two possible values of the quantity $r_{\nu}$, namely $r_{0}=\left|\mathbf{r}_{0}\right|=$ $\left|\mathbf{r}-\mathbf{r}_{A}\right|=\sqrt{x^{2}+y^{2}+(z-d)^{2}}$ and $r_{1}=\left|\mathbf{r}_{1}\right|=\left|\mathbf{r}-\mathbf{r}_{I}\right|=$ $\sqrt{x^{2}+y^{2}+(z+d)^{2}}$, where $\mathbf{r}_{I} \equiv(0,0,-d)$ is the position of the image of the atom reflected on the wall. $r_{0}$ is the distance of the observation point $\mathbf{r}=(x, y, z)$ from the atom in $\mathbf{r}_{A} \equiv(0,0, d)$, whereas $\mathbf{r}_{I} \equiv(0,0,-d)$ is its distance from the atom's image.

The final step for the evaluation of the first-order correction is the evaluation of the $k$-integral. Using (6) and the following integral representations of the Heaviside step function $\theta(x)$

$$
\begin{aligned}
& \int_{-\infty}^{\infty} d k \frac{1-e^{ \pm i\left(k_{0}-k\right) c t}}{k-k_{0}} \sin \left(k r_{\nu}\right)=\pi e^{ \pm i k_{0} r_{\nu}} \theta\left(c t-r_{\nu}\right) \\
& \int_{-\infty}^{\infty} d k \frac{1-e^{i\left(k \pm k_{0}\right) c t}}{k \pm k_{0}} \cos \left(k r_{\nu}\right)=-i \pi e^{ \pm i k_{0} r_{\nu}} \theta\left(c t-r_{\nu}\right)
\end{aligned}
$$

we obtain the following expressions for the first-order correction of the electric and magnetic field operators generated by the two-level atom in front of a perfectly conducting wall

$$
\begin{aligned}
& E_{p}^{(1)}(\mathbf{r}, t)=\sum_{q} \sum_{\nu=0}^{1}(-1)^{\nu\left(1-\delta_{q z}\right)} \mu_{q} F_{p q} \frac{1}{r_{\nu}}\left[S_{+} e^{i k_{0}\left(c t-r_{\nu}\right)}+S_{-} e^{-i k_{0}\left(c t-r_{\nu}\right)}\right] \theta\left(c t-r_{\nu}\right) \\
& H_{p}^{(1)}(\mathbf{r}, t)=-\sum_{q} \sum_{\nu=0}^{1}(-1)^{\nu\left(1-\delta_{q z}\right)} \mu_{q} G_{p q} \frac{1}{r_{\nu}} \frac{\partial}{\partial r_{\nu}}\left[S_{+} e^{i k_{0}\left(c t-r_{\nu}\right)}+S_{-} e^{-i k_{0}\left(c t-r_{\nu}\right)}\right] \theta\left(c t-r_{\nu}\right) .
\end{aligned}
$$

We wish to stress that expressions (13) are general operatorial relations, which can be used for calculating matrix elements between any pair of atom-field states.

It is worthy analyzing from a physical point of view 
the expressions obtained. The sum over $\nu$ in (13) describes the existence of two distinct contributions to these operators. The first one depends on the parameter $r_{0}=\left|\mathbf{r}-\mathbf{r}_{A}\right|$, that is the distance between the observation point $\mathbf{r}$ and the atom. We shall refer to it as the direct term. The second one is a function of $r_{1}=\left|\mathbf{r}-\mathbf{r}_{I}\right|$, that is the distance between the observation point and the image atom. We will call it the reflected term. Both terms propagate satisfying rigorously the causality principle as shown by the presence of the $\theta$ functions. We can check that our results (13) correctly yield the known analogous results for an atom in the free space as obtained in [9], if we take the limiting case of the wall very distant from the atom: in fact, in this limit $r_{0}$ is finite, whereas $r_{1}$ grows with the atom-wall distance. In the same limit we can thus keep only the $\nu=0$ term in (13), and obtain the same expressions found in [9]. A final remark concerning the action of the differential operators in (13) is necessary, because in principle they should be applied to the Heaviside functions also. This means that the Dirac delta function and its derivatives show up, yielding a singular impulse propagating as a wave front on the light cone, strongly dependent on the model used (in particular on the dipole approximation). Similar singular behaviour were found in the field emitted during the dynamical dressing of an atom [13, 16, 20]. In the applications we will discuss in the following Sections we will not consider the electromagnetic field on this (singular) wave front and so we will not explicitly evaluate it (hence the results in the next Section will not be valid for $t=r_{0} / c$ or $\left.t=r_{1} / c\right)$.

We now consider the second-order correction to the electric and magnetic field operators, obtained by substitution of the third of (5) into (3). Since the calculation is quite straightforward, although lengthy, we shall only give the final results. In the expressions obtained, two independent sums over wavevectors and polarizations are present: one of them can be explicitly performed and finally yields a $\theta$ function ensuring relativistic causality. Thus we get

$$
\begin{gathered}
E_{p}^{(2)}(\mathbf{r}, t)=-2 i S_{z} \sqrt{\frac{2 \pi c}{\hbar V}} \sum_{\mathbf{k} j} \sqrt{k}\left(\boldsymbol{\mu} \cdot \mathbf{f}_{\mathbf{k} j}\left(\mathbf{r}_{A}\right)\right) \sum_{q} \sum_{\nu=0,1}(-1)^{\nu\left(1-\delta_{q z}\right)} \mu_{q} F_{p q} \frac{1}{r_{\nu}} \\
\times\left\{a_{\mathbf{k} j}\left[\frac{1}{\omega_{0}+\omega_{\mathbf{k}}}\left(e^{-i k\left(c t-r_{\nu}\right)}-e^{i k_{0}\left(c t-r_{\nu}\right)}\right)+\frac{1}{\omega_{0}-\omega_{\mathbf{k}}}\left(e^{-i k\left(c t-r_{\nu}\right)}-e^{-i k_{0}\left(c t-r_{\nu}\right)}\right)\right]+\right. \\
\left.\quad-a_{\mathbf{k} j}^{\dagger}\left[\frac{1}{\omega_{0}+\omega_{\mathbf{k}}}\left(e^{i k\left(c t-r_{\nu}\right)}-e^{-i k_{0}\left(c t-r_{\nu}\right)}\right)+\frac{1}{\omega_{0}-\omega_{\mathbf{k}}}\left(e^{i k\left(c t-r_{\nu}\right)}-e^{i k_{0}\left(c t-r_{\nu}\right)}\right)\right]\right\} \theta\left(c t-r_{\nu}\right) \\
H_{p}^{(2)}(\mathbf{r}, t)=2 i S_{z} \sqrt{\frac{2 \pi c}{\hbar V}} \sum_{\mathbf{k} j} \sqrt{k}\left(\boldsymbol{\mu} \cdot \mathbf{f}_{\mathbf{k} j}\left(\mathbf{r}_{A}\right)\right) \sum_{q} \sum_{\nu=0,1} \mu_{q}(-1)^{\nu\left(1-\delta_{q z}\right)} G_{p q} \frac{1}{r_{\nu}} \\
\frac{\partial r_{\nu}}{2}\left\{a_{\mathbf{k} j}\left[\frac{1}{\omega_{0}+\omega_{\mathbf{k}}}\left(e^{-i k\left(c t-r_{\nu}\right)}-e^{i k_{0}\left(c t-r_{\nu}\right)}\right)+\frac{1}{\omega_{0}-\omega_{\mathbf{k}}}\left(e^{-i k\left(c t-r_{\nu}\right)}-e^{-i k_{0}\left(c t-r_{\nu}\right)}\right)\right]\right. \\
\left.-a_{\mathbf{k} j}^{\dagger}\left[\frac{1}{\omega_{0}+\omega_{\mathbf{k}}}\left(e^{i k\left(c t-r_{\nu}\right)}-e^{-i k_{0}\left(c t-r_{\nu}\right)}\right)+\frac{1}{\omega_{0}-\omega_{\mathbf{k}}}\left(e^{i k\left(c t-r_{\nu}\right)}-e^{i k_{0}\left(c t-r_{\nu}\right)}\right)\right]\right\} \theta\left(c t-r_{\nu}\right) .
\end{gathered}
$$

The second-order fields in (14), similarly to the firstorder ones (13), show the presence of a direct and a reflected term.

\section{ELECTRIC AND MAGNETIC ENERGY DENSITIES OF THE FIELD}

In this Section we shall use the results of Section IIIfor calculating the electric and magnetic field energy densities generated by the atom when the plate is present; they are of course related to the field fluctuations generated by the atom. In the next Section the results obtained will be applied to evaluate the dynamical Casimir-Polder potential between two atoms placed near the conducting plate (in the so-called far zone).
We shall first evaluate the square of the electric field operator and its average value on the initial state we are mostly interested in. The square of the electric field operator coincides with the electric energy density operator, apart a factor $1 / 8 \pi$, and also with the electric field fluctuations, assuming a vanishing expectation value of the electric field. Using the expansion of the electric field up to the second order, we have

$$
\begin{aligned}
& E^{2}(\mathbf{r}, t)=\mathbf{E}^{(0)}(\mathbf{r}, t) \cdot \mathbf{E}^{(0)}(\mathbf{r}, t) \\
& \quad+\left(\mathbf{E}^{(0)}(\mathbf{r}, t) \cdot \mathbf{E}^{(1)}(\mathbf{r}, t)+\mathbf{E}^{(1)}(\mathbf{r}, t) \cdot \mathbf{E}^{(0)}(\mathbf{r}, t)\right) \\
& \quad+\left(\mathbf{E}^{(1)}(\mathbf{r}, t) \cdot \mathbf{E}^{(1)}(\mathbf{r}, t)+\mathbf{E}^{(0)}(\mathbf{r}, t) \cdot \mathbf{E}^{(2)}(\mathbf{r}, t)\right. \\
& \left.\quad+\mathbf{E}^{(2)}(\mathbf{r}, t) \cdot \mathbf{E}^{(0)}(\mathbf{r}, t)\right)+\ldots
\end{aligned}
$$


The first term is a free-field term that does not depend on the presence of the source; then we have first- and second-order terms. From now onwards we consider the specific initial atom-field state $\left|0_{\mathbf{k} j}, \downarrow_{A}\right\rangle$, where the field is in its vacuum state and the "source" atom (A) is in its bare ground state, and calculate the (time-dependent) expectation value of (15). This quantity gives the time evolution of the electric energy density during the selfdressing process of atom A, that in the next Section will be used to evaluate the dynamical Casimir-Polder interaction energy between two atoms when the conducting plate is present.

The average value of the zeroth-order contribution is independent of the presence of the atom and thus does not contribute to atom-atom interactions (it gives the time-independent but spatially dependent fluctuations of the electric field with the plate present and in the absence of the atom). Also, the first-order term has a zero mean value on our initial state. Thus, the first relevant non-vanishing contributions are given by the secondorder terms. We start evaluating the average value of $\mathbf{E}^{(1)}(\mathbf{r}, t) \cdot \mathbf{E}^{(1)}(\mathbf{r}, t)$

$$
\begin{aligned}
& \left\langle 0_{\mathbf{k} j}, \downarrow_{A}\left|\mathbf{E}^{(1)}(\mathbf{r}, t) \cdot \mathbf{E}^{(1)}(\mathbf{r}, t)\right| 0_{\mathbf{k} j}, \downarrow_{A}\right\rangle \\
& \quad=\sum_{p q q^{\prime}} \sum_{\nu \nu^{\prime}=0,1} \mu_{q} \mu_{q^{\prime}}(-1)^{\nu\left(1-\delta_{q z}\right)}(-1)^{\nu^{\prime}\left(1-\delta_{q^{\prime} z}\right)} F_{p q}\left(\frac{e^{i k_{0} r_{\nu}}}{r_{\nu}} \theta\left(c t-r_{\nu}\right)\right) F_{p q^{\prime}}\left(\frac{e^{-i k_{0} r_{\nu^{\prime}}}}{r_{\nu^{\prime}}} \theta\left(c t-r_{\nu^{\prime}}\right)\right)
\end{aligned}
$$

where $k_{0}=\omega_{0} / c$. As discussed in Section III, we do not to apply the differential operators to the $\theta$ functions, because this would only add singular terms on the light cone, in which we are not interested at the moment. Using the result

$$
F_{p q} \frac{e^{i k r}}{r}=k^{3} f_{p q}(k r) e^{i k r}
$$

where

$$
f_{p q}(k r)=\frac{\delta_{p q}-\hat{r}_{p} \hat{r}_{q}}{k r}+\left(\delta_{p q}-3 \hat{r}_{p} \hat{r}_{q}\right)\left(\frac{i}{k^{2} r^{2}}-\frac{1}{k^{3} r^{3}}\right)
$$

we obtain

$$
\begin{aligned}
\left\langle 0_{\mathbf{k} j}, \downarrow_{A}\left|\mathbf{E}^{(1)}(\mathbf{r}, t) \cdot \mathbf{E}^{(1)}(\mathbf{r}, t)\right| 0_{\mathbf{k} j}, \downarrow_{A}\right\rangle= & -\sum_{\nu \nu^{\prime}=0,1} k_{0}^{6} \mu_{q} \mu_{q^{\prime}}(-1)^{\nu\left(1-\delta_{q z}\right)}(-1)^{\nu^{\prime}\left(1-\delta_{q^{\prime} z}\right)} f_{p q}\left(k_{0} r_{\nu}\right) f_{p q^{\prime}}\left(-k_{0} r_{\nu^{\prime}}\right) \\
& \times e^{i k_{0}\left(r_{\nu}-r_{\nu^{\prime}}\right)} \theta\left(c t-r_{\nu}\right) \theta\left(c t-r_{\nu^{\prime}}\right) .
\end{aligned}
$$

We can analyze the previous expression in three different space-time regions. When $c t<r_{0}$, that is for times shorter than the time taken by a light signal to travel from the atom in $\mathbf{r}_{A}$ to the observation point $\mathbf{r}$, it is zero because both Heaviside functions are vanishing. For $r_{0}<c t<r_{1}$, i.e. after that a light signal can travel from the atom to the observation point but before this point has been reached by the signal reflected on the wall, only the contribution with $\nu=\nu^{\prime}=0$ is different from zero and the result is the same obtained in 12 for an atom in the free space. For $c t>r_{1}$, that is after that even the reflected signal has passed the observation point, all terms contribute, and we can recognize a contribution from the atom $\left(\nu=\nu^{\prime}=0\right)$, one from the image atom $\left(\nu=\nu^{\prime}=1\right)$ and also interference terms $\left(\nu \neq \nu^{\prime}\right)$. The result in free space is recovered by taking the limit $r_{0}<<r_{1}$ (i.e. very distant wall). Similar consideration holds for the other contributions we are now going to evaluate.

In the continuum limit, the contribution from second-order fields in (15) becomes

$$
\begin{aligned}
& \left\langle 0_{\mathbf{k} j}, \downarrow_{A}\left|\mathbf{E}^{(0)}(\mathbf{r}, t) \cdot \mathbf{E}^{(2)}(\mathbf{r}, t)\right| 0_{\mathbf{k} j}, \downarrow_{A}\right\rangle=-\frac{1}{\pi} \sum_{p q q^{\prime}} \sum_{\nu \nu^{\prime}=0,1} \mu_{q} \mu_{q^{\prime}}(-1)^{\nu\left(1-\delta_{q z}\right)}(-1)^{\nu^{\prime}\left(1-\delta_{q^{\prime} z}\right)} \theta\left(c t-r_{\nu}\right) \\
& \quad \times \int_{0}^{+\infty} d k e^{-i k c t} F_{p q} \frac{1}{r_{\nu}}\left[\frac{1}{k+k_{0}}\left(e^{-i k_{0}\left(c t-r_{\nu}\right)}-e^{i k\left(c t-r_{\nu}\right)}\right)+\frac{1}{k_{0}-k}\left(e^{i k_{0}\left(c t-r_{\nu}\right)}-e^{i k\left(c t-r_{\nu}\right)}\right)\right] F_{p q^{\prime}}\left[\frac{\sin \left(k r_{\nu^{\prime}}\right)}{r_{\nu^{\prime}}}\right] .
\end{aligned}
$$


Finally, after some straightforward algebra, we obtain

$$
\begin{aligned}
\left\langle 0_{\mathbf{k} j}, \downarrow_{A}\right. & \left.\left|\mathbf{E}^{(0)}(\mathbf{r}, t) \cdot \mathbf{E}^{(2)}(\mathbf{r}, t)\right| 0_{\mathbf{k} j}, \downarrow_{A}\right\rangle+\left\langle 0_{\mathbf{k} j}, \downarrow_{A}\left|\mathbf{E}^{(2)}(\mathbf{r}, t) \cdot \mathbf{E}^{(0)}(\mathbf{r}, t)\right| 0_{\mathbf{k} j}, \downarrow_{A}\right\rangle \\
= & -\frac{1}{2 \pi i} \sum_{p, q, q^{\prime}} \sum_{\nu, \nu^{\prime}=0}^{1} \int_{0}^{+\infty} d k k^{3} \mu_{q} \mu_{q^{\prime}}(-1)^{\nu\left(1-\delta_{q z}\right)}(-1)^{\nu^{\prime}\left(1-\delta_{q^{\prime} z}\right)} \theta\left(c t-r_{\nu}\right) \\
& \times\left[\frac { 1 } { k _ { 0 } + k } \left(k_{0}^{3} f_{p q}\left(k_{0} r_{\nu}\right) f_{p q^{\prime}}\left(k r_{\nu^{\prime}}\right) e^{-i k_{0}\left(c t-r_{\nu}\right)} e^{-i k\left(c t-r_{\nu^{\prime}}\right)}+\right.\right. \\
& \left.+k_{0}^{3} f_{p q}\left(k_{0} r_{\nu}\right) f_{p q^{\prime}}\left(-k r_{\nu^{\prime}}\right) e^{-i k_{0}\left(c t-r_{\nu}\right)} e^{-i k\left(c t+r_{\nu^{\prime}}\right)}+k^{3} f_{p q}\left(-k r_{\nu}\right) f_{p q^{\prime}}\left(-k r_{\nu^{\prime}}\right) e^{-i k\left(r_{\nu}+r_{\nu^{\prime}}\right)}\right) \\
& +\frac{1}{k_{0}-k}\left(-k_{0}^{3} f_{p q}\left(-k_{0} r_{\nu}\right) f_{p q^{\prime}}\left(k r_{\nu^{\prime}}\right) e^{i k_{0}\left(c t-r_{\nu}\right)} e^{-i k\left(c t-r_{\nu^{\prime}}\right)}\right. \\
& \left.\left.-k_{0}^{3} f_{p q}\left(-k_{0} r_{\nu}\right) f_{p q^{\prime}}\left(-k r_{\nu^{\prime}}\right) e^{i k_{0}\left(c t-r_{\nu}\right)} e^{-i k\left(c t+r_{\nu^{\prime}}\right)}+k^{3} f_{p q}\left(-k r_{\nu}\right) f_{p q^{\prime}}\left(-k r_{\nu^{\prime}}\right) e^{-i k\left(r_{\nu}+r_{\nu^{\prime}}\right)}\right)\right]+ \text { c.c. }
\end{aligned}
$$

where the terms with a pole in $k=k_{0}$ should be taken as their principal value. We now extend the integral over $k$ to the complex plane, closing the integration path at infinity in the fourth quadrant and on the negative imaginary axis. Assuming no other singularity in the integrand function except the pole at $k=k_{0}$ and that it vanishes at infinity, the result can be written as an integral along the imaginary axis plus the pole contribution

$$
P \int_{0}^{\infty} d k g(k)=-i \int_{0}^{\infty} d k g(-i k)-i \pi \operatorname{Res}\{g(k)\}_{k=k_{0}}
$$

We now give the explicit expression of the expectation value of the square of the electric field for $t>r_{1} / c$, that is after that the direct and reflected waves have reached the observation point (this quantity vanishes for $t<r_{0} / c$, of course, but it is not zero for $r_{0} / c<t<r_{1} / c$ due to the "direct" contribution). In this timescale, the contribution from the pole at $k=k_{0}$ for $t>r_{1} / c$ in (22) exactly cancels the contribution (19) when the three second-order contributions in (15) are summed up, exactly as in the case of an atom in the free space discussed in [12]. We obtain

where $g(k)$ indicates the integrand function.

$$
\begin{aligned}
\left\langle 0_{\mathbf{k} j}, \downarrow_{A}\left|E^{2}(\mathbf{r}, t)\right| 0_{\mathbf{k} j}, \downarrow_{A}\right\rangle & -E_{\mathrm{zp}}^{E}=-\frac{\hbar c}{\pi} \sum_{p q q^{\prime}} \sum_{\nu \nu^{\prime}=0}^{1}(-1)^{\nu\left(1-\delta_{q z}\right)}(-1)^{\nu^{\prime}\left(1-\delta_{q^{\prime} z}\right)} \\
& \times\left[\int_{0}^{\infty} d k k^{6} \alpha_{q q^{\prime}}(i k) f_{p q}\left(i k r_{\nu}\right) f_{p q^{\prime}}\left(i k r_{\nu^{\prime}}\right) e^{-k\left(r_{\nu}+r_{\nu^{\prime}}\right)}\right. \\
& -\frac{i k_{0}^{2}}{2} \int_{0}^{\infty} d k k^{3} e^{-k c t} \alpha_{q q^{\prime}}(i k)\left(k_{0} \operatorname{Re} f_{p q}\left(k_{0} r_{\nu}\right) e^{-i k_{0}\left(c t-r_{\nu}\right)}-k \operatorname{Im} f_{p q}\left(k_{0} r_{\nu}\right) e^{-i k_{0}\left(c t-r_{\nu}\right)}\right) \\
& \left.\times\left(f_{p q^{\prime}}\left(-i k r_{\nu^{\prime}}\right) e^{k r_{\nu^{\prime}}}+f_{p q^{\prime}}\left(i k r_{\nu^{\prime}}\right) e^{-k r_{\nu^{\prime}}}\right)\right]
\end{aligned}
$$

where we have explicitly indicated that the "zero-point" electric energy density $E_{\mathrm{zp}}^{E}$ due to zero-order field operators has been subtracted, and we have also introduced the dynamic atomic polarizability tensor at imaginary frequencies

$$
\alpha_{q q^{\prime}}(i k)=\frac{2 k_{0} \mu_{q} \mu_{q^{\prime}}}{\hbar c\left(k^{2}+k_{0}^{2}\right)}
$$

It is worth considering the limiting case of Eq. (23) for $t \rightarrow+\infty$. In this limit, fast oscillating terms inside the $k$-integral average to zero, finally yielding the time-independent value given by the first two lines only of Eq. (23). For an isotropic atom or polarizable body $\left(\mu_{x}=\mu_{y}=\mu_{z}\right)$ this becomes 


$$
\begin{aligned}
& \left\langle 0_{\mathbf{k} j}, \downarrow_{A}\left|E^{2}(\mathbf{r})\right| 0_{\mathbf{k} j}, \downarrow_{A}\right\rangle-E_{\mathrm{zp}}^{E}=\frac{2 \hbar c}{\pi} \sum_{\nu=0}^{1} \int_{0}^{+\infty} d k k^{6} \alpha(i k)\left(\frac{1}{k^{2} r_{\nu}^{2}}+\frac{2}{k^{3} r_{\nu}^{3}}+\frac{5}{k^{4} r_{\nu}^{4}}+\frac{6}{k^{5} r_{\nu}^{5}}+\frac{3}{k^{6} r_{\nu}^{6}}\right) e^{-2 k r_{\nu}} \\
& +\frac{2 \hbar c}{\pi} \sum_{p q}(-1)^{1-\delta_{q z}} \int_{0}^{+\infty} d k k^{6} \alpha(i k)\left[\frac{\delta_{p q}-\hat{r}_{0 p} \hat{r}_{0 q}}{k r_{0}}+\left(\delta_{p q}-3 \hat{r}_{0 p} \hat{r}_{0 q}\right)\left(\frac{1}{k^{2} r_{0}^{2}}+\frac{1}{k^{3} r_{0}^{3}}\right)\right] \\
& \times\left[\frac{\delta_{p q}-\hat{r}_{1 p} \hat{r}_{1 q}}{k r_{1}}+\left(\delta_{p q}-3 \hat{r}_{1 p} \hat{r}_{1 q}\right)\left(\frac{1}{k^{2} r_{1}^{2}}+\frac{1}{k^{3} r_{1}^{3}}\right)\right] e^{-k\left(r_{0}+r_{1}\right)} .
\end{aligned}
$$

where $\alpha(k)$ is the isotropic electric polarizability of the atom. There are three different terms that contribute to the electric energy density. The first comes from the $\nu=\nu^{\prime}=0$ contribution and it is identical to that found in 12] for a two-level atom in the free space. The second term is the $\nu=\nu^{\prime}=1$ contributions and has the same functional expression of the previous one with $r_{0}$ replaced by $r_{1}$ (distance of the observation point from the image of the atom). Finally, there is a mixed term depending on both distances $r_{0}$ and $r_{1}$. The physical meaning and role on interatomic Casimir-Polder interactions of these terms will be discussed in detail in the next Section.

Similarly to the electric case, from the results of Section III we can calculate the magnetic energy density, which we shall use in the next Section in order to calculate magnetic interatomic Casimir forces in the far zone. We shall not give the explicit expressions of the timedependent magnetic energy density, but only its asymptotic expression for $t \rightarrow+\infty$. In this limit it settles to a time-independent value, as the electric energy density, given by

$$
\begin{aligned}
\left\langle 0_{\mathbf{k} j}, \downarrow_{A}\left|H^{2}(\mathbf{r})\right| 0_{\mathbf{k} j}, \downarrow_{A}\right\rangle-E_{\mathrm{zp}}^{M}=\frac{2 k_{0}}{\pi} \sum_{p, q, q^{\prime}} \sum_{\nu, \nu^{\prime}=0,1} \mu_{q} \mu_{q^{\prime}}(-1)^{\nu\left(1-\delta_{q z}\right)}(-1)^{\nu^{\prime}\left(1-\delta_{q^{\prime} z}\right)} \\
\quad \times \int_{0}^{+\infty} d k \frac{k^{6}}{k_{0}^{2}+k^{2}} g_{p q}\left(i k r_{\nu}\right) g_{p q^{\prime}}\left(i k r_{\nu^{\prime}}\right) e^{-k\left(r_{\nu}+r_{\nu^{\prime}}\right)} \\
=\frac{\hbar c}{\pi} \sum_{p, q, q^{\prime}} \sum_{\nu, \nu^{\prime}=0,1}(-1)^{\nu\left(1-\delta_{q z}\right)}(-1)^{\nu^{\prime}\left(1-\delta_{q^{\prime} z}\right)} \int_{0}^{+\infty} d k k^{6} \alpha_{q q^{\prime}}(i k) g_{p q}\left(i k r_{\nu}\right) g_{p q^{\prime}}\left(i k r_{\nu^{\prime}}\right) e^{-k\left(r_{\nu}+r_{\nu^{\prime}}\right)}
\end{aligned}
$$

(similarly to the electric case, the zero-point magnetic energy density has been subtracted) where

$$
g_{p q}(i k r)=i \sum_{s} \epsilon_{p q s} \hat{r}_{s}\left(\frac{1}{k r}+\frac{1}{k^{2} r^{2}}\right) .
$$

For an isotropic molecule it becomes

$$
\left\langle 0_{\mathbf{k} j}, \downarrow_{A}\left|H^{2}(\mathbf{r})\right| 0_{\mathbf{k} j}, \downarrow_{A}\right\rangle-E_{\mathrm{zp}}^{M}=\frac{\hbar c}{\pi} \sum_{p, q} \sum_{\nu, \nu^{\prime}=0,1}(-1)^{\left(\nu+\nu^{\prime}\right)\left(1-\delta_{q z}\right)} \int_{0}^{+\infty} d k k^{6} \alpha(i k) g_{p q}\left(i k r_{\nu}\right) g_{p q}\left(i k r_{\nu^{\prime}}\right) e^{-k\left(r_{\nu}+r_{\nu^{\prime}}\right)}
$$

\section{THE FAR-ZONE ELECTRIC AND MAGNETIC INTERATOMIC CASIMIR-POLDER ENERGIES WITH BOUNDARY CONDITIONS}

In this Section we apply the results of the previous Sections for the time-dependent Maxwell fields to the calculation of dynamical electric and magnetic CasimirPolder interactions between two atoms in the presence of the conducting wall, also recovering known results in the asymptotic regime. The electric and magnetic field oper- ators of Section III, and the related expectation values of the energy densities obtained in Section IV, can be used to obtain static and dynamical Casimir-Polder forces between two atoms in the presence of a boundary condition such as a conducting wall. In fact, the interatomic Casimir-Polder interaction in the so-called far zone, that is for distances between the atoms larger than their typical transition wavelength from the ground state, can be obtained from the interaction of one atom, described as a polarizable body, with the field fluctuations generated 
by the other atom [21, 22, 23]. This approach has been recently proved to be useful also in the case of dynamical (time-dependent) Casimir-Polder forces for atoms in excited states [14] or in partially dressed states [13]. Thus we evaluate the (electric) Casimir-Polder interaction energy in the far zone between our "source" atom A and a second atom $B$ placed in $\mathbf{r}_{B}$, when the conducting wall in present, as

$$
\Delta E_{E}=-\frac{1}{2} \alpha_{E}^{B}\left\langle E^{2}\left(\mathbf{r}_{B}, t\right)\right\rangle_{A}
$$

where $\alpha_{E}^{B}$ is the static electric polarizability of atom B, assumed isotropic for simplicity, and the average value of $E^{2}(\mathbf{r}, t)$, evaluated at the position of atom $\mathrm{B}$, is calculated on the initial state of the field and of atom A (for example the bare ground state, as in the previous Section). In a quasi-static approach, the Casimir-Polder force is then obtained as minus the derivative of the interaction energy with respect to the distance between the two atoms. Effects related to the atomic motion, as discussed in [15, 17], are neglected here. Besides being well suited for dealing with dynamical interactions, our method also allows a simpler derivation of static CasimirPolder forces and a transparent physical interpretation, compared to the usual methods based on fourth-order perturbation calculations [24].

From our results dynamical magnetic Casimir-Polder interactions can also be easily obtained, such as the interaction of a magnetically polarizable body B (with static magnetic polarizability $\alpha_{B}^{M}$ ) with the magnetic field fluctuations generated by atom $\mathrm{A}$, as

$$
\Delta E_{M}=-\frac{1}{2} \alpha_{M}^{B}\left\langle H^{2}\left(\mathbf{r}_{B}, t\right)\right\rangle_{A}
$$

The relations above show that the Casimir-Polder interaction energy between two atoms is proportional to the expectation value of the square of the fields generated by one of the two atoms at the position of the other atom, that is to the average electric (and magnetic) energy density or equivalently to the field fluctuations generated by the atom.

In order to evaluate equation (29) in the far zone, we can use the expression (25) of the electric energy density with $\mathbf{r}=\mathbf{r}_{B}$ (in the limit $t \rightarrow \infty$ ), approximated by substituting the dynamical polarizability of the atom with the static one; this allows to perform the $k$ integral. We finally obtain

$$
\begin{aligned}
\Delta E_{E}= & -\frac{1}{2} \alpha_{E}^{B}\left\langle E^{2}\left(\mathbf{r}_{B}\right)\right\rangle_{A}=-\frac{23 \hbar c}{4 \pi} \alpha_{E}^{A} \alpha_{E}^{B}\left(\frac{1}{r_{0}^{7}}+\frac{1}{r_{1}^{7}}\right) \\
& +\frac{16 \hbar c \alpha_{E}^{A} \alpha_{E}^{B}}{\pi r_{0}^{3} r_{1}^{3}\left(r_{0}+r_{1}\right)^{5}}\left[3 r_{0}^{2} r_{1}^{2}+\rho^{2}\left(r_{0}^{2}+5 r_{0} r_{1}+r_{1}^{2}\right)\right]
\end{aligned}
$$

with

$$
\rho=r_{0} \sin \theta_{0}=r_{1} \sin \theta_{1}
$$

where $\theta_{0}\left(\theta_{1}\right)$ is the angle between the vector $\mathbf{r}_{0}\left(\mathbf{r}_{1}\right)$ and the perpendicular to the wall.

Expression (31), which was already obtained with different methods in [3, 4], shows that in the presence of the conducting plate, the Casimir-Polder interaction between the two atoms $\mathrm{A}$ and $\mathrm{B}$ consists of three terms: the $r_{0}^{-7}$ interaction between the two atoms as in the absence of the wall; the $r_{1}^{-7}$ interaction between one atom and the image of the other atom as reflected by the wall; a term involving both coordinates $r_{0}$ and $r_{1}$. The first two terms give an attractive component, whereas the third one gives a repulsive component. However, as discussed in [4], the total interaction is attractive for any geometric configuration of the atoms with respect to the wall.

Our results in the previous Section also allow to evaluate the time-dependent Casimir-Polder interaction between the two atoms, using the time-dependent expression for the squared electric energy density (23) (and not only its asymptotic value for $t \rightarrow \infty$ ). The interaction energy between the two atoms in the far zone as a function of time for $c t>r_{1}$, that is after that both the direct and reflected fields generated by atom $\mathrm{A}$ have reached atom $\mathrm{B}$, is characterized by oscillations around its asymptotic value, with an amplitude decaying with time to zero. For $c t \gg r_{1}$ the energy settles to its stationary value, as obtained in a time-independent approach [4] or from (31). This behavior is analogous to that already found in the time-dependent Casimir-Polder force between an atom and a conducting wall [16].

In a similar way we can explicitly evaluate the magnetic far-zone Casimir-Polder interaction energy, in the presence of the wall, by substituting (28) into (30). In the far zone, after substitution of the dynamical polarizability of the atom with the static one and the $k$ integration we get

$$
\begin{aligned}
\Delta E_{M} & =-\frac{1}{2} \alpha_{M}^{B}\left\langle H^{2}\left(\mathbf{r}_{B}\right)\right\rangle_{A}=\frac{7 \hbar c}{4 \pi} \alpha_{E}^{A} \alpha_{M}^{B}\left(\frac{1}{r_{0}^{7}}+\frac{1}{r_{1}^{7}}\right) \\
& -\frac{16 \hbar c \alpha_{E}^{A} \alpha_{M}^{B} r_{0} \cos \theta_{0} r_{1} \cos \theta_{1}}{\pi r_{0}^{3} r_{1}^{3}\left(r_{0}+r_{1}\right)^{5}}\left[r_{0}^{2}+5 r_{0} r_{1}+r_{1}^{2}\right] .
\end{aligned}
$$

We note that, similarly to the electric case, the magnetic interaction contains three terms: two are related to the atom-atom (depending from $r_{0}$ only) and atomimage (depending from $r_{1}$ only) interactions, and the other one depends on both coordinates $r_{0}$ and $r_{1}$. It should be also noted that the magnetic Casimir-Polder interaction between the two atoms is repulsive. However, in ordinary situations magnetic Casimir-Polder interactions are much weaker than electric ones, the total force being attractive. For large atom-wall distance $\left(r_{1} \gg r_{0}\right)$, Eq. (33) reduces to the well-know stationary electric-magnetic Casimir-Polder interaction between two atoms in free space [25, 26]. Finally, we wish to stress that the atom-atom dynamical Casimir-Polder interactions discussed in this Section add, of course, to the known dynamical atom-wall interaction energies, discussed in [15, 16, 17] for the case of electric interactions. 


\section{CONCLUSIONS}

In this paper we have calculated the time-dependent electric and magnetic Maxwell field operators in the Heisenberg representation for a two-level atomic source near a boundary condition such as a perfectly conducting wall. The method used consists of an iterative solution of the Heisenberg equations for the field operators. The results obtained for the field operators are quite general, not depending on the specific atom-field initial state. We have explicitly obtained solutions up to the second order in the electric charge and showed that they can be separated into a direct and reflected term. The latter is related to the presence of the wall. Both terms propagate in a strictly causal way. We have then evaluated the time-dependent electric and magnetic energy densities around the atom for a specific initial state of the system, that is the atom in its bare ground state and the field in its vacuum state. This has finally allowed us to evaluate the dynamical (time-dependent) Casimir-Polder interaction energy between the "source" atom and a second atom, considered as a polarizable body, when both are placed in the vicinity of the conducting wall. Both electric and magnetic Casimir-Polder energies have been considered. We have also shown that for $t \rightarrow \infty$ both the electric and magnetic dynamical Casimir-Polder energies settle to time-independent values, which coincide with those that can be obtained by a time-independent approach. We stress that our method is general and not limited to a specific initial state of the system; in fact our solutions for the field operators in the presence of the wall can be used for any atom-field initial state, being operator solutions.

\section{APPENDIX: POLARIZATION SUM}

In this Appendix we evaluate the following expressions which have been used in Section III.

$$
\begin{aligned}
& \sum_{j} \int d \Omega\left(\mathbf{f}_{\mathbf{k} j}(\mathbf{r})\right)_{p}\left(\mathbf{f}_{\mathbf{k} j}\left(\mathbf{r}_{A}\right)\right)_{q} \\
& \sum_{j} \int d \Omega\left(\nabla \times \mathbf{f}_{\mathbf{k} j}(\mathbf{r})\right)_{p}\left(\mathbf{f}_{\mathbf{k} j}\left(\mathbf{r}_{A}\right)\right)_{q}
\end{aligned}
$$

where $p, q=x, y, z$. In order to show the method used, we first make a specific choice of $p$ and $q$, namely $p=x$ and $q=y$, and we calculate the first expression of (A.1. The technique is the same for all the other cases. Using the mode functions (2) and applying the sum rule for the polarization unit vectors

$$
\sum_{j}\left(\hat{e}_{\mathbf{k} j}\right)_{p}\left(\hat{e}_{\mathbf{k} j}\right)_{q}=\delta_{p q}-\hat{k}_{p} \hat{k}_{q}
$$

where $\delta_{p q}$ is the Kronecker delta function, we get

$$
\begin{aligned}
& \sum_{j} \int d \Omega\left(\mathbf{f}_{\mathbf{k} j}(\mathbf{r})\right)_{x}\left(\mathbf{f}_{\mathbf{k} j}\left(\mathbf{r}_{A}\right)\right)_{y} \\
& =-\frac{8}{k^{2}} \int d \Omega k_{x} k_{y} \cos \left[k_{x}\left(x+\frac{L}{2}\right)\right] \sin \left(k_{x} L / 2\right) \\
& \quad \times \sin \left[k_{y}\left(y+\frac{L}{2}\right)\right] \cos \left(k_{y} L / 2\right) \sin \left(k_{z} z\right) \sin \left(k_{z} d\right) .
\end{aligned}
$$

We can now exploit the fact that in the limit $L \rightarrow+\infty$, the oscillating functions containing $L$ can be replaced by their mean value over a period; for example

$$
\begin{aligned}
\sin ^{2}\left(k_{x} L / 2\right) & \rightarrow 1 / 2 \\
\sin \left(k_{x} L / 2\right) \cos \left(k_{x} L / 2\right) & \rightarrow 0 .
\end{aligned}
$$

Thus we get

$$
\begin{aligned}
& \frac{2 k_{x} k_{y}}{k^{2}} \sin \left(k_{x} x\right) \sin \left(k_{y} y\right) \sin \left(k_{z} z\right) \sin \left(k_{z} d\right) \\
& =\frac{2}{k^{2}} \nabla_{x} \nabla_{y}\left[\cos \left(k_{x} x\right) \cos \left(k_{y} y\right) \sin \left(k_{z} z\right) \cos \left(k_{z} d\right)\right] .
\end{aligned}
$$

Finally, using

$$
\int d \Omega \cos \left(k_{x} a\right) \cos \left(k_{y} b\right) \cos \left(k_{z} c\right)=4 \pi \frac{\sin (k D)}{k D}
$$

with $D=\sqrt{a^{2}+b^{2}+c^{2}}$, we get

$$
\sum_{j} \int d \Omega\left(\mathbf{f}_{\mathbf{k} j}(\mathbf{r})\right)_{x}\left(\mathbf{f}_{\mathbf{k} j}\left(\mathbf{r}_{A}\right)\right)_{y}=\frac{4 \pi}{k^{3}} \sum_{\nu=0,1} \nabla_{x} \nabla_{y} \frac{\sin \left(k r_{\nu}\right)}{r_{\nu}}
$$

where $r_{0}=\sqrt{x^{2}+y^{2}+(z-d)^{2}}$ and $r_{1}=$ $\sqrt{x^{2}+y^{2}+(z+d)^{2}}$. All the other terms can be obtained using the same method. The general results are

$$
\begin{aligned}
& \sum_{j} \int d \Omega\left(\mathbf{f}_{\mathbf{k} j}(\mathbf{r})\right)_{p}\left(\mathbf{f}_{\mathbf{k} j}\left(\mathbf{r}_{A}\right)\right)_{q} \\
& \quad=\frac{4 \pi}{k^{3}} \sum_{\nu=0}^{1}(-1)^{\nu\left(1-\delta_{q z}\right)} F_{p q} \frac{\sin \left(k r_{\nu}\right)}{r_{\nu}}
\end{aligned}
$$

and

$$
\begin{aligned}
& \sum_{j} \int d \Omega\left(\nabla \times \mathbf{f}_{\mathbf{k} j}(\mathbf{r})\right)_{p}\left(\mathbf{f}_{\mathbf{k} j}\left(\mathbf{r}_{A}\right)\right)_{q} \\
& =\frac{4 \pi}{k} \sum_{\nu=0}^{1}(-1)^{\nu\left(1-\delta_{q z}\right)} G_{p q} \frac{\sin \left(k r_{\nu}\right)}{r_{\nu}}
\end{aligned}
$$

where $F_{p q}$ and $G_{p q}$ are the differential operators defined in Section III 


\section{ACKNOWLEDGMENTS}

R.V. acknowledges Finnish CIMO and Turun Yliopistosäätiö for financial support. R.M. and R.P. ac- knowledge partial financial support from Ministero dell'Università e della Ricerca Scientifica e Tecnologica and by Comitato Regionale di Ricerche Nucleari e di Struttura della Materia.
[1] P. W. Milonni, The Quantum Vacuum: An Introduction to Quantum Electrodynamics (Academic Press, San Diego, 1994)

[2] G. Compagno, R. Passante, and F. Persico, Atom-Field Interactions and Dressed Atoms (Cambridge University Press, Cambridge, 1995)

[3] E. A. Power and T. Thirunamachandran, Phys. Rev. A 25, 2473 (1982)

[4] S. Spagnolo, R. Passante, and L. Rizzuto, Phys. Rev. A 73, 062117 (2006)

[5] Y. Sherkunov, Phys. Rev. A 72, 052703 (2005)

[6] M. Markovitch and H. Diamant, Phys. Rev. Lett. 95, 223203 (2005)

[7] Y. Sherkunov, Phys. Rev. A 75, 012705 (2007)

[8] M. S. Tomas, Phys. Rev. A 75, 012109 (2007)

[9] E. A. Power and T. Thirunamachandran, Phys. Rev. A 28, 2663 (1983)

[10] E. A. Power and T. Thirunamachandran, Phys. Rev. A 28, 2671 (1983)

[11] E. A. Power and T. Thirunamachandran, Phys. Rev. A 47, 2539 (1993)

[12] E. A. Power and T. Thirunamachandran, Phys. Rev. A 45, 54 (1992)

[13] R. Passante and F. Persico, Phys. Lett. A 312, 319 (2003)

[14] L. Rizzuto, R. Passante, and F. Persico, Phys. Rev. A 70, 012107 (2004)
[15] S. Shresta, B. L. Hu, and N. G. Phillips, Phys. Rev. A 68, 062101 (2003)

[16] R. Vasile and R. Passante, Phys. Rev. A 78, 032108 (2008)

[17] B. L. Hu, A. Roura, and S. Shresta, J. Opt. B: Quantum Semiclass. Opt. 6, S698 (2004)

[18] E. A. Power and S. Zineau, Phil. Trans. Roy. Soc. A 251, 427 (1959)

[19] E. A. Power and T. Thirunamachandran, Phys. Rev. A 28, 2649 (1983)

[20] G. Compagno, R. Passante, and F. Persico, Phys. Rev. A 38, 600 (1988)

[21] R. Passante and E. A. Power, Phys. Rev. A 35, 188 (1987)

[22] D. P. Craig and E. A. Power, Int. J. Quantum Chem. 3, 903 (1969)

[23] R. Passante, E. A. Power, and T. Thirunamachandran, Phys. Lett. A 249, 77 (1998)

[24] D. P. Craig and T. Thirunamachandran, Molecular Quantum Electrodynamics (Dover Publ., Mineola, NY 1998)

[25] G. Feinberg and J. Sucher, J. Chem. Phys. 48, 3333 (1968)

[26] G. Feinberg and J. Sucher, Phys. Rev. A 2, 2395 (1970) 